\title{
A USER'S GUIDE TO FORTRAN PROGRAMS FOR WIGNER AND RACAH COEFFICIENTS OF $\mathrm{SU}_{3} *$
}

\author{
Yoshimi AKIYAMA ** and J.P. DRAAYER \\ Dep. of Physics, The University of Michigan, Ann Arbor, Michigan 48104, USA \\ Received 16 February 1973
}

PROGRAM SUMMARY

Title of program: SU3 WIGNER \& RACAH COEFFICIENTS

Cotalogue number: ACRM

Computer: IBM 360/67; Installation: The University of Michigan, Ann Arbor, Michigan, USA

Operating system: MTS $/ 360$

Programming language used: FORTRAN IV

High speed storage required: $\mathrm{SU}_{3} \supset \mathrm{SU}_{2} \times \mathrm{U}_{1}$ Wigner coefficients, 13008 words

$\mathrm{SU}_{3}$ Racah coefficients, 14654 words;

$\mathrm{SU}_{3} \supset \mathrm{R}_{3}$ Wigner coefficients, 14202 words

$\mathrm{SU}_{3}$ Racah coefficients, 14654 words. $\mathrm{SU}_{3} \supset \mathrm{R}_{3}$ Wigner coefficients, 14202 words

No. of bits in a word: 32

Is the program overlaid? No.

No. of magnetic tapes required: None.

Other peripherals used: Card reader, line printer

No. of cards in combined program and test deck: 2046

Keywords: $\mathrm{SU}_{3}$, Wigner coefficient, Racah coefficient, Clebsch-Gordan coefficient, Recoupling coefficient, Isoscalar factor, U-function, Unitary coupling, Unitary recoupling, K-band projection, Hypercharge.

Nature of physical problem

$\mathrm{SU}_{3} \supset \mathrm{SU}_{2} \times \mathrm{U}_{1}$ and $\mathrm{SU}_{3} \supset \mathrm{R}_{3}$ Wigner coefficients as well as $\mathrm{SU}_{3}$ Racah coefficients are calculated for arbitrary couplings and multiplicity.

\section{Method of solution}

A build-up process based on the Biedenharn-Louck prescription for specifying the outer multiplicity is employed to generate $\mathrm{SU}_{3} \supset \mathrm{SU}_{2} \times \mathrm{U}_{1}$ Wigner coefficients [1]. $\mathrm{SU}_{3}$ Racah coefficients follow through standard recoupling formulae [2]. $\mathrm{SU}_{3} \supset \mathrm{R}_{3}$ Wigner coefficients are obtained from the corresponding $\mathrm{SU}_{3} \supset \mathrm{SU}_{2} \times \mathrm{U}_{1}$ Wigner coefficients via unitary transformation coefficients relating $\mathrm{SU}_{3} \supset \mathrm{SU}_{2} \times \mathrm{U}_{1}$ and $\mathrm{SU}_{3} \supset \mathrm{R}_{3}$ basis states [3].

* Work supported by the U.S. National Science Foundation.

** Present address: Department of Physics, Nikon University, Tokyo, Japan.
Restrictions on the complexity of the problem

Factorials $M !, M<M_{\max }=32$, and binomial coefficients

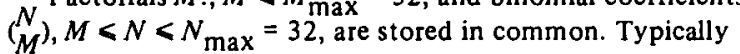
for $S_{3} \supset S_{2} \times U_{1}$ Wigner coefficients $\Lambda_{1}+\Lambda_{2}+\Lambda_{3}<M_{\max }$ whereas for $\mathrm{SU}_{3} \supset \mathrm{R}_{3}$ Wigner coefficients $\lambda+\mu+L<N_{\max }$. The limits $M_{\max }$ and $N_{\max }$ may be altered by modifying one and only one subprogram.

References

[1] J.P. Draayer and Yoshimi Akiyama J. Math. Phys, in press.

[2] K.T. Hecht, Nuclear Physics 62 (1965) 1.

[3] J.P. Draayer, Nuclear Physics A129 (1969) 647. 